\title{
Adherence to recommendations of Barrett's esophagus surveillance guidelines: a systematic review and meta-analysis
}

Authors

Carlijn A. M. Roumans ${ }^{1,2}$, Ruben D. van der Bogt ${ }^{1}$, Ewout W. Steyerberg ${ }^{2,3}$, Dimitris Rizopoulos ${ }^{4}$, Iris LansdorpVogelaar ${ }^{2}$, Prateek Sharma ${ }^{5,6}$, Manon C. W. Spaander ${ }^{1,{ }^{*}}$, Marco J. Bruno ${ }^{1,{ }^{*}}$

Institutions

1 Department of Gastroenterology and Hepatology, Erasmus MC University Medical Center, Rotterdam, The Netherlands

2 Department of Public Health, Erasmus MC University Medical Center, Rotterdam, The Netherlands

3 Department of Medical Statistics and Bioinformatics, Leiden University Medical Center, Leiden, The Netherlands

4 Department of Biostatistics, Erasmus MC University Medical Center, Rotterdam, The Netherlands

5 Department of Gastroenterology and Hepatology, University of Kansas Medical Center, Kansas City, Kansas, United States

6 Department of Gastroenterology and Hepatology, Veterans Affairs Medical Center, Kansas City, Missouri, United States

submitted 1.4.2019

accepted after revision 15.7.2019

Bibliography

DOI https://doi.org/10.1055/a-0995-0134

Published online: 17.9.2019 | Endoscopy 2020; 52: 17-28

(c) Georg Thieme Verlag KG Stuttgart · New York

ISSN 0013-726X

Corresponding author

Manon C. W. Spaander, MD, PhD, Department of

Gastroenterology and Hepatology (room Na-610), Erasmus

MC University Medical Center, P.O. Box 2040, 3000 CA

Rotterdam, The Netherlands

Fax: +31-10-7035643

v.spaander@erasmusmc.nl

Tables 1s-6s, Figs. 1s-3s, supplementary references Online content viewable at:

https://doi.org/10.1055/a-0995-0134

Scan this QR-Code for the author commentary.

\section{ABSTRACT}

Background Guidelines aim to reduce treatment variation and improve quality of care. In the literature there is large variation in the reported rates of adherence to recommendations of surveillance for Barrett's esophagus (BE). The aim of this systematic review was to identify explanatory parameters determining these differences in adherence rates.

Methods Embase, Medline Epub, and Web of Science were searched. Studies reporting adherence in at least one of five domains were selected: general domain, surveillance interval, biopsy protocol, landmark identification, and histopathological information. Adherence was expressed as the proportion of endoscopies or endoscopists being in accordance with guideline recommendations. Variation in adherence was evaluated by 1 ) meta-regression of adherence rates in random effects meta-analysis to define subgroups, and 2) compiling an overview of the most reported explanatory parameters for (non)adherence.

Results 56 studies, including 14002 BE patients and 4932 endoscopists, were included. Subgroup analysis showed that variation in rates of adherences to surveillance interval recommendations ( $\left.R^{2}=98 \%-99 \%\right)$ was explained by difference in country (43\%), by practice type ( $90 \%)$, and by year of publication (11\%). Variation in adherence to the Seattle protocol was explained by difference in country (14\%). Factors most frequently reported to be associated with better adherence were shorter BE length, salaried employment, surveillance in university hospitals, and dedicated programs.

Conclusions This study provides insight into the variability of rates of adherence to BE surveillance recommendations between studies. Better adherence in university hospitals and dedicated programs indicate that persistent alertness of guidelines is important.

\footnotetext{
* These authors contributed equally to this work.
} 


\section{Introduction}

Barrett's esophagus (BE) is a premalignant condition of esophageal adenocarcinoma (EAC), and is associated with gastroesophageal reflux disease. The incidence of EAC has been rising over past decades. In the advanced stage, morbidity and mortality of EAC are high, with a dismal prognosis [1]. Surveillance was introduced to detect early signs of neoplastic progression. Studies have shown repeatedly that BE patients participating in a surveillance program had EAC detected at an earlier stage compared with sporadic (symptomatic) EAC patients [2]. In addition to a better prospect of survival, early detection also offers the possibility to apply minimally invasive treatment, such as endoscopic eradication therapy, in selected cases. The generally accepted recommendation for patients with BE is to undergo regular endoscopic follow-up with biopsies according to the Seattle protocol.

In general, guidelines aim to reduce treatment variation and improve quality of care [3-6]. Adherence to guideline recommendations for surveillance of BE has been shown to be low, with varying consequences [7]. In particular, if impaired costeffectiveness of surveillance due to shorter surveillance intervals is accompanied by lower detection of dysplasia because of nonadherence to the Seattle protocol and the Prague classification, the healthcare burden of surveillance of BE patients is disproportionate [8]. This may result in increased costs for society and poorer detection of early neoplastic progression in BE patients [9].

The key question is, Why is the gap between guideline recommendations and practice patterns so substantial in studies? As well as low rates of adherence to guideline recommendations, high adherence rates have also been reported [10]. Sources of this between-study variance are unclear. Explanatory factors for adherence may also explain this heterogeneity in adherence rates between studies. Insight into the reason(s) behind adherence may identify areas for improvement.

In this systematic review and meta-analysis we aimed to identify explanatory parameters of (non)adherence by exploring the sources of variation between studies and determining risk factors for (non)adherence as reported in the literature.

\section{Methods}

This systematic literature review and meta-analysis was based on the Preferred Reporting Items for Systematic Reviews and Meta-Analyses (PRISMA) statement.

\section{Search strategy}

An electronic search in Embase, Medline Epub (Ovid), and Web of Science was performed without restrictions in date or language on 3 July 2017. The search strategy included the terms relating to Barrett's esophagus, adherence to the guideline, and surveillance (see Table $1 \mathrm{~s}$ in the online-only supplementary material). Bibliographies of full-text articles assessed for eligibility were handsearched.

\section{Study selection}

Five domains within BE surveillance were defined, based on guidelines: general domain, surveillance interval, biopsy protocol, landmark identification, and histopathological information. Within these domains, 19 single guideline recommendations were selected ( $\triangleright$ Table $\mathbf{1}$ ). The recommended surveillance interval, Seattle protocol, and Prague classification were assigned as primary recommendations because of the expected importance in BE surveillance. Adherence to all of these recommendations was estimated. To estimate adherence to the Prague classification, only studies conducted after 2006 were included, as this was the year of publication of this classification [11].

Studies reporting adherence to at least one of the 19 recommendations were selected. Studies conducted in the setting of a hospital, freestanding or open-access endoscopy center, or based on the registry of members of a gastroenterological association, were assessed for inclusion. Participants in these studies had to be adults with known BE, with or without histological confirmation, who were enrolled in a surveillance program, or endoscopists performing BE surveillance. No minimum number of participants or duration of follow-up was set for studies to be considered for inclusion. Types of studies eligible for this review were cross-sectional, retrospective, and prospective cohort studies. Conference abstracts were included only if the data were not published in peer-reviewed full-text format.

Studies of patients who had already reached the end point of high grade dysplasia, EAC, or who had undergone previous endoscopic therapy were excluded. If studies only focused on the effect of an intervention to improve adherence, such as a dedicated surveillance program, they were included if baseline data were available in the same or previously published article or abstract.

Records identified by this search strategy were initially screened, after deduplication, on title and abstract by one author (C.A.M.R.), and relevant full-text articles were then selected. A second author (R.D.vdB) reviewed this process. In cases of disagreement, a third independent author (M.C.W.S.) was consulted. All reviewers were working in the field of gastroenterology in both clinical practice and research. The deduplication and management of records was performed using EndNote X7.5 reference manager (Thomson Reuters).

\section{Data extraction and synthesis}

The extraction form used to collect the study data was based on the Strengthening the Reporting of Observational studies in Epidemiology (STROBE) statement.

The primary outcome was the variability between studies explained by a specific subgroup, expressed as $R^{2}$. This represents the variance in adherence rates, as explained for example by difference in type of practice between studies, expressed as a percentage. To be able to determine this between-study variance, we extracted data concerning adherence to guideline recommendations in individual studies. We defined adherence as a proportion of the number of adherent participants divided by the total number of participants. As subjects could be either 
- Table 1 Guideline recommendations and associated patient, endoscopist, and facility variables, as scored for adherence in this systematic review and meta-analysis.

\begin{tabular}{|c|c|}
\hline Domain & Recommendation in the guideline \\
\hline \multirow[t]{2}{*}{ General } & Performance of any form of surveillance \\
\hline & Total adherence to the guideline \\
\hline \multirow[t]{3}{*}{ Surveillance interval } & Surveillance interval in general* \\
\hline & Surveillance interval in patients without dysplasia (NDBE)* \\
\hline & Surveillance interval in patients with low grade dysplasia* \\
\hline \multirow[t]{2}{*}{ Biopsy protocol } & Seattle protocol* \\
\hline & Use of advanced imaging/high definition/high resolution white-light endoscopy \\
\hline \multirow[t]{9}{*}{ Landmark identification } & Report of indication of endoscopy \\
\hline & Report of level of squamocolumnar junction \\
\hline & Report of level of gastroesophageal junction \\
\hline & Report of level of diaphragm impression \\
\hline & Report of presence of hiatal hernia \\
\hline & Report of length of BE using Prague criteria* \\
\hline & Report of length of BE in general \\
\hline & Report of presence of inflammation \\
\hline & Report of presence of visible abnormalities \\
\hline \multirow[t]{3}{*}{ Histopathological information } & Report of presence of intestinal metaplasia \\
\hline & Report of presence of dysplasia \\
\hline & Review of second pathologist in cases of suspected dysplasia \\
\hline Field of association & Factors of potential association with adherence \\
\hline \multirow[t]{4}{*}{ Patient variables } & Age \\
\hline & Sex \\
\hline & Ethnicity \\
\hline & Length of $B E$ \\
\hline \multirow[t]{9}{*}{ Endoscopist variables } & Age \\
\hline & Sex \\
\hline & Years in practice \\
\hline & Number of EGDs per week or month \\
\hline & Reimbursement \\
\hline & Belief in efficacy \\
\hline & Surgeon or physician \\
\hline & Awareness of the guideline \\
\hline & Agreement with the guideline \\
\hline Facility variables & Type of practice \\
\hline Other & Effect of intervention to improve adherence \\
\hline
\end{tabular}


patients with BE in a study based on data retrieved from endoscopy and pathology reports, or endoscopists in a questionnaire-based study, we referred to them as participants. If only the practice pattern was reported (e.g. the number of endoscopies with an interval of 3 years in nondysplastic patients, rather than the number of adherent endoscopies), these results were compared with the guideline as mentioned in the article. If no guideline was mentioned, recommendations of the most recently issued guideline at the time and location of that publication were used to determine the proportion of adherence [3-5,12-29]. In the United States, where multiple guidelines exist, such cases (i.e. only the practice pattern was reported in a study from the USA, but no guideline mentioned), the guideline of the American College of Gastroenterology was used.

The secondary outcome was to search for associations between adherence rates and explanatory parameters that had already been determined in the studies, with regard to patient, endoscopist, or facility variables. The direction of the association (increased or decreased probability of adherence), and the presence or absence of its statistical significance, were extracted.

\section{Quality assessment}

Publication bias was assessed separately for each recommendation by funnel plots. In the process of methodological quality assessment of the risk of bias in individual studies, another tool was used for each outcome.

Because adherence to the guideline recommendations was considered a proportion, the methodological quality of studies was evaluated using the Loney Scoring Tool, adjusted to our systematic review. Studies estimating prevalence or incidence of a certain condition can be scored in this tool at a scale from 0 (worst score) to 8 (best score). A score of $\geq 5$ was considered good quality.

The quality of methods for associated factors was assessed using the Quality In Prognosis Studies tool (QUIPS) [30]. Each aspect could be scored as low, moderate, or high risk of bias.

\section{Statistical analysis}

In order to calculate between-study variance, first the proportions of adherence of individual studies were pooled in a random effects meta-analysis with a log odds transformation to stabilize data. In cases where the proportion of adherence was zero, a continuity correction was applied. This pooled estimate and $95 \%$ confidence interval $(\mathrm{Cl})$ was interpreted as the proportion of adherent participants. Heterogeneity was estimated using Cochran's test ( $Q$ statistic) and $R^{2}$ statistic with a threshold value of $25 \%, 50 \%$, and $75 \%$ for low, moderate, and high, respectively [31]. Only adherence reported as a proportion with absolute numbers could be used in the meta-analysis. Next, for primary recommendations (surveillance interval, Seattle protocol, Prague classification), possible sources of heterogeneity were investigated in a subgroup analysis. We used univariate meta-regression analysis. Prespecified variables that were tested were year of publication and country; post hoc we added type of practice and data collection (i.e. either self-reported surveys or data retrieved from endoscopy and/or pa- thology reports). In the subgroup type of practice, Veterans Affairs medical facilities were considered as community hospitals, and BE centers and teaching hospitals were considered as university hospitals. The difference between subgroups was reported as an odds ratio (OR). The variability between studies explained by these subgroups was expressed using $R^{2}$.

In addition to using the exploration of between-study variance as a potential source of parameters explaining (non)adherence, the associations between adherence rates and explanatory parameters that had already been determined in the included studies were evaluated. Only those explanatory parameters that determined adherence rates to at least two primary recommendations (surveillance interval, Seattle protocol, Prague classification) were plotted separately in a figure. Metaanalysis was not possible because of the limited number of studies per association. If statistical significance was not reported in the studies, the chi-squared test with continuity correction was used where possible to calculate statistical significance of the association.

Statistical calculations were done using R, version 3.4.1 ( $R$ Foundation for Statistical Computing, Vienna, Austria; www.Rproject.org), using the metafor package for meta-analysis. $P$ values of $<0.05$ were considered statistically significant.

\section{Sensitivity analysis}

Outliers were identified based on a plot of influence diagnostics outliers. Sensitivity analyses by excluding these studies showed the difference compared with the original results. In addition, the effect of excluding studies with a score of methodological quality $\leq 5$ was evaluated.

\section{Results}

\section{Study selection and characteristics}

Initially 684 records were identified. After deduplication, 475 were screened on title and abstract. Out of 86 articles assessed for eligibility, 60 articles containing 56 studies were included for qualitative and 49 articles containing 45 studies for quantitative analyses. In some articles, the same study was conducted with a different sample size, which explains the difference in number of articles and studies included ( $\mathbf{F i g} \mathbf{1} \mathbf{1}$ ).

The 56 studies (Table 2s) could be subdivided into four categories based on type of participants and methods used. In 19 cross-sectional studies, gastroenterologists and surgeons practicing surveillance were surveyed by a questionnaire to determine their practice patterns and factors influencing adherence. In 26 retrospective cohort studies (17 single-center and nine multicenter studies), endoscopy and pathology reports of $\mathrm{BE}$ patients under surveillance were reviewed. In six prospective studies, the effect of an intervention to improve adherence rates was evaluated. The other five studies were a combination of the previously mentioned, or other designs.

Of all studies included, the setting was reported as follows: 9 community hospitals and 16 university hospitals; 20 studies reported data collection from a combination of these types of centers, and in 11 studies the type of practice was unclear. The earliest study was published in 1997, the most recent in 2017. 


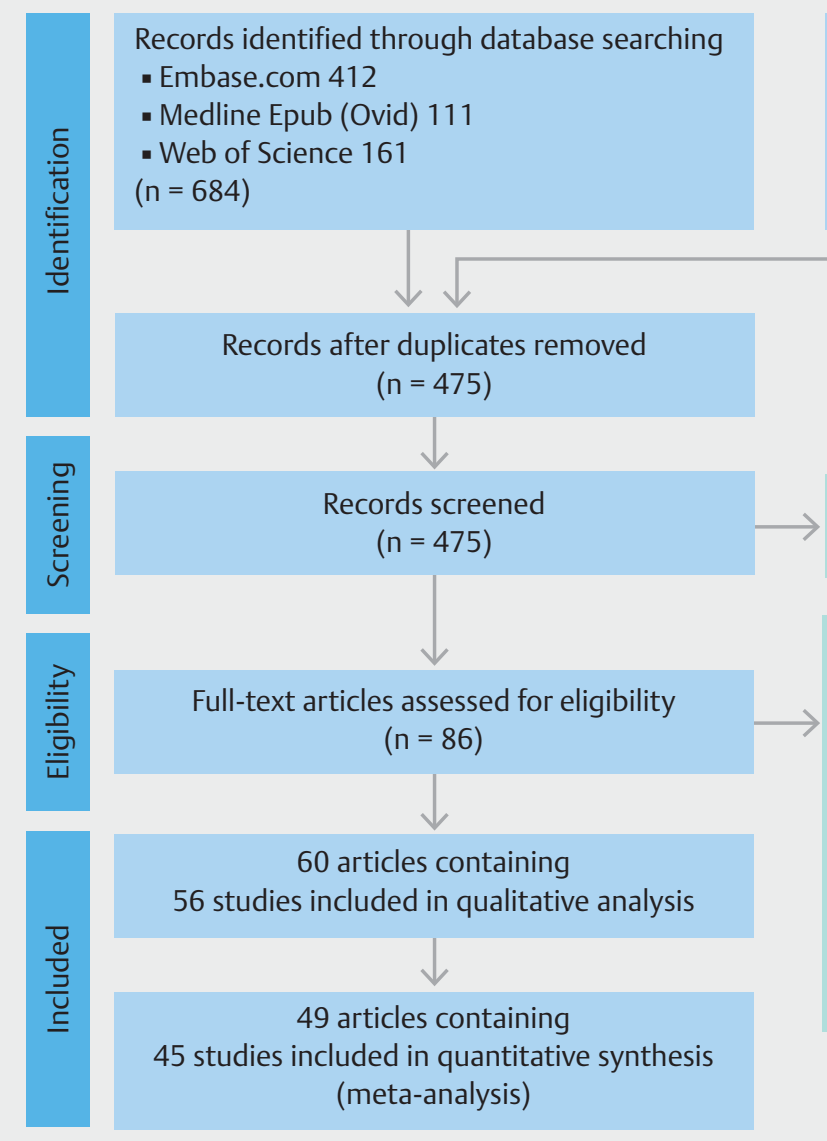

Additional records identified through other sources $(n=9)$

Records excluded $(n=389)$

Full-text articles excluded $(\mathbf{n}=\mathbf{2 6}$ )

- Comment on other study (9)

- Focus on effectiveness of surveillance (6)

- Update of guideline (3)

- Qualitative study of adherence (3)

- Focus on associated factors (1)

- Interview expert (1)

- Inconsistencies (1)

- Only effect intervention available (1)

- Data not in accordance with our aim (1)

- Fig. 1 Study flow diagram.

The geographical setting was developed countries in all studies: Argentina, Australia, several countries in Europe, and North America. A total of $14002 \mathrm{BE}$ patients were included, with a median sample size of 210 (interquartile range [IQR] 103 367), and 4932 endoscopists participated, with a median sample size of 203 (IQR $123-260)$.

Data from the original papers were not included in the analysis in cases of: 1) lack of report of numbers, results were only described in words (four studies) [supplementary references $47,50,73,97]$; 2) report of mean surveillance interval, mean number of biopsies, or mean biopsy percentage, instead of proportions (eight studies) [supplementary references 42, 51, 54, $58,70,84,87,96]$; 3) separate reporting of adherence to fourquadrant biopsies and biopsies every $2 \mathrm{~cm}$ (three studies) [supplementary references $58,60,67]$; and 4 ) separate reporting of adherence in participating centers (one study) [supplementary reference 50$]$.

\section{Subgroups explaining between-study variance}

Pooled proportions of adherence ranged from $18 \%$ to $89 \%$ ( $\triangleright$ Fig.2). For recommendations with pooled estimates based on $\geq 20$ studies, forest plots were constructed ( $\triangleright$ Fig. 3, Fig. 1s, Fig. 2s). Adherence to surveillance interval was $55 \%$ for nondysplastic Barrett's esophagus (NDBE) (95\%Cl 44\%-66\%), $50 \%$ for low grade dysplasia (LGD) (95\%Cl $38 \%-62 \%)$, and $49 \%$ to the Seattle protocol ( $95 \% \mathrm{Cl} 36-62 \%)$. In Table 3s, the details of other pooled estimates are shown.

In addition to providing information concerning the proportion of adherence, which was pooled in the meta-analysis, several studies also showed whether intervals were too short or too long. For the recommendation "interval NDBE," 13 studies reported an interval that was too short [supplementary references $41,47,51,53,60,62,67,70,77,78,90,91,95]$, and only one study reported an interval that was too long among the nonadherent participants [supplementary reference 56]; one study reported an equal proportion of endoscopies with surveillance intervals that were too short or too long [supplementary reference 74]. For “interval LGD," 12 studies reported an interval that was too short [supplementary references 41,47 , $53,60,62,67,75,77,88,90,91,95]$, and in four studies the interval was too long [supplementary references 70, 56, 74, 78]. For the recommendation "Seattle protocol," 12 studies reported that fewer biopsies than expected were taken [supplementary references $42,54,56,58,70,73-75,84,91,95,96]$, and one study reported a surplus [supplementary reference 77 ].

Heterogeneity was high ( $\geq 75 \%$ ) for all pooled estimates. For "surveillance interval general," $90 \%$ of heterogeneity could be explained by difference in type of practice between studies 

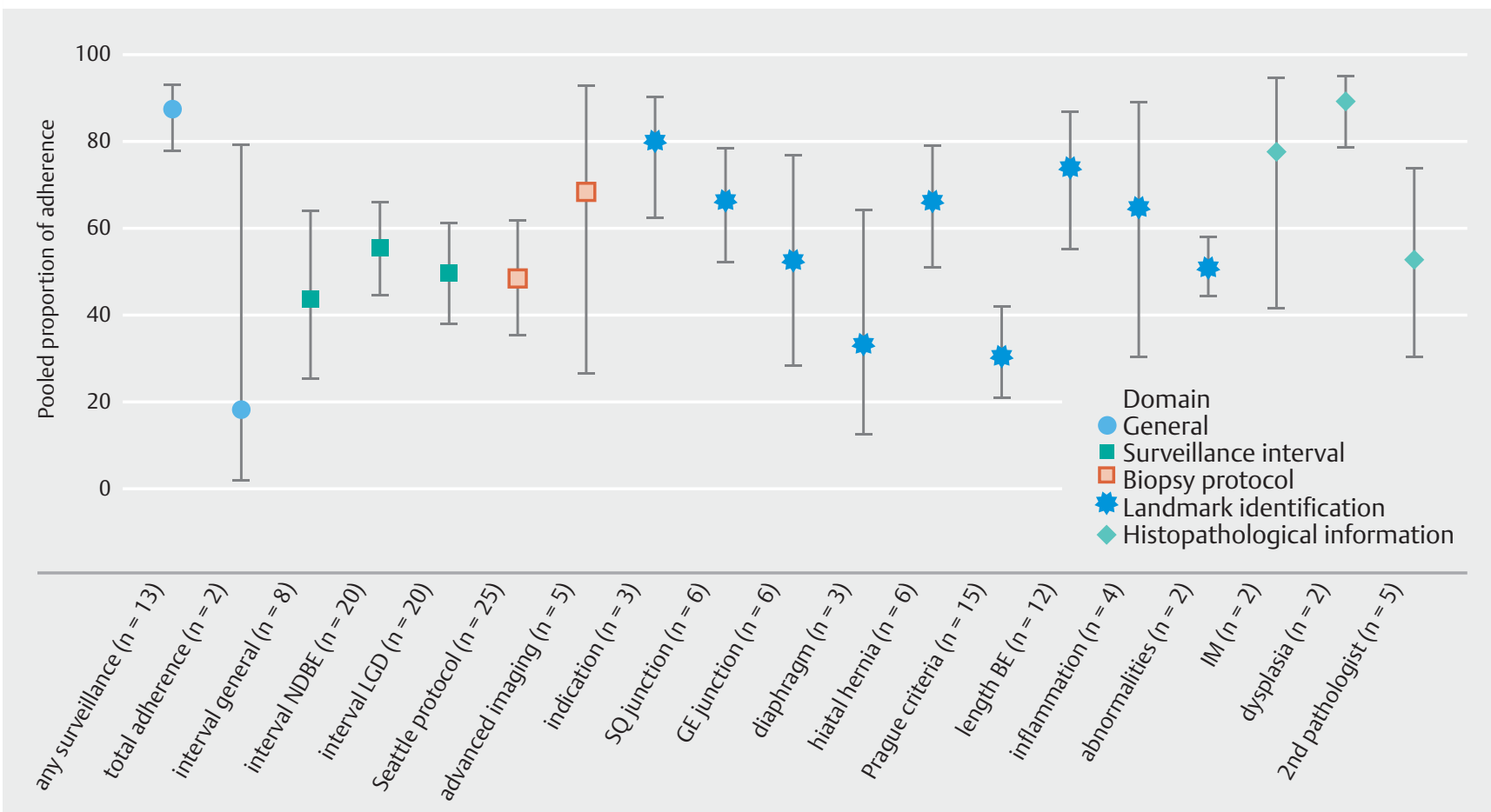

- Fig. 2 Summary of pooled proportions and $95 \%$ confidence intervals of adherence to guideline recommendations, subdivided per domain. n, number of studies included in the meta-analysis; BE, Barrett's esophagus; GE, gastroesophageal; IM, intestinal metaplasia; LGD, low grade dysplasia; NDBE, nondysplastic Barrett's esophagus; SQ, squamocolumnar.

and $11 \%$ by year of publication ( $\triangleright$ Table 2 ). For “interval NDBE," $43 \%$ could be explained by differences in country. For "Seattle protocol," $14 \%$ of the between-study variance was explained by differences in country. And for the "Prague classification," $12 \%$ of heterogeneity was due to differences in data collection.

\section{Factors associated with surveillance guideline adherence}

An overview was compiled of reported factors that influence adherence to primary recommendations of the surveillance guideline ( $\triangleright$ Fig.4). The following factors were reported most frequently to be associated with better adherence: shorter BE segments (Seattle protocol); salaried employment instead of productivity-based employment of the endoscopists (surveillance intervals); university hospitals (Seattle protocol and Prague classification); the introduction of dedicated surveillance programs, often reported as "interventions" (surveillance intervals, Seattle protocol, and Prague classification). The type of interventions reported to improve adherence were participation in a trial, pooling patients on a dedicated list, and formalized and multifaceted intervention programs. Only the implementation and dissemination of guidelines were not reported as often to improve adherence to guideline recommendations. Other factors less frequently reported to be associated with better adherence to guideline recommendations were more belief in the efficacy of surveillance, and awareness and agreement of the endoscopists with the guideline (surveillance interval), younger age of the endoscopists (surveillance interval and Seattle pro- tocol), and physicians compared with surgeons (Prague criteria). Only 10 out of 29 studies performed multivariable analysis to identify factors associated with better adherence.

\section{Quality assessment}

Methodological quality concerning the between-study variance was good for 34 studies (scored $\geq 5$, Table 4s), while 15 scored < 5 . Biased data collection using self-reported surveys, inadequate response rate, and a lack of adequate description of participants led to reduced quality scores most frequently. Studies with a low score on aspect 5 of the Loney Scoring tool also scored low on aspect 4 of the QUIPS (Table 5s) within the context of the methodological quality of the secondary objective. The maximum score was low for 1 study, medium for 14 studies, and high for 15 studies. Reduced quality was often attributable to the lack of correction for confounders and self-reported outcome measurement. Funnel plots were rather symmetric, providing no evidence of publication bias (Fig. 3s).

\section{Sensitivity analysis}

The exclusion of outliers or low quality studies showed similar results in assessing adherence rates. The results of sensitivity analyses of adherence to guideline recommendations are shown in Table 6s. 


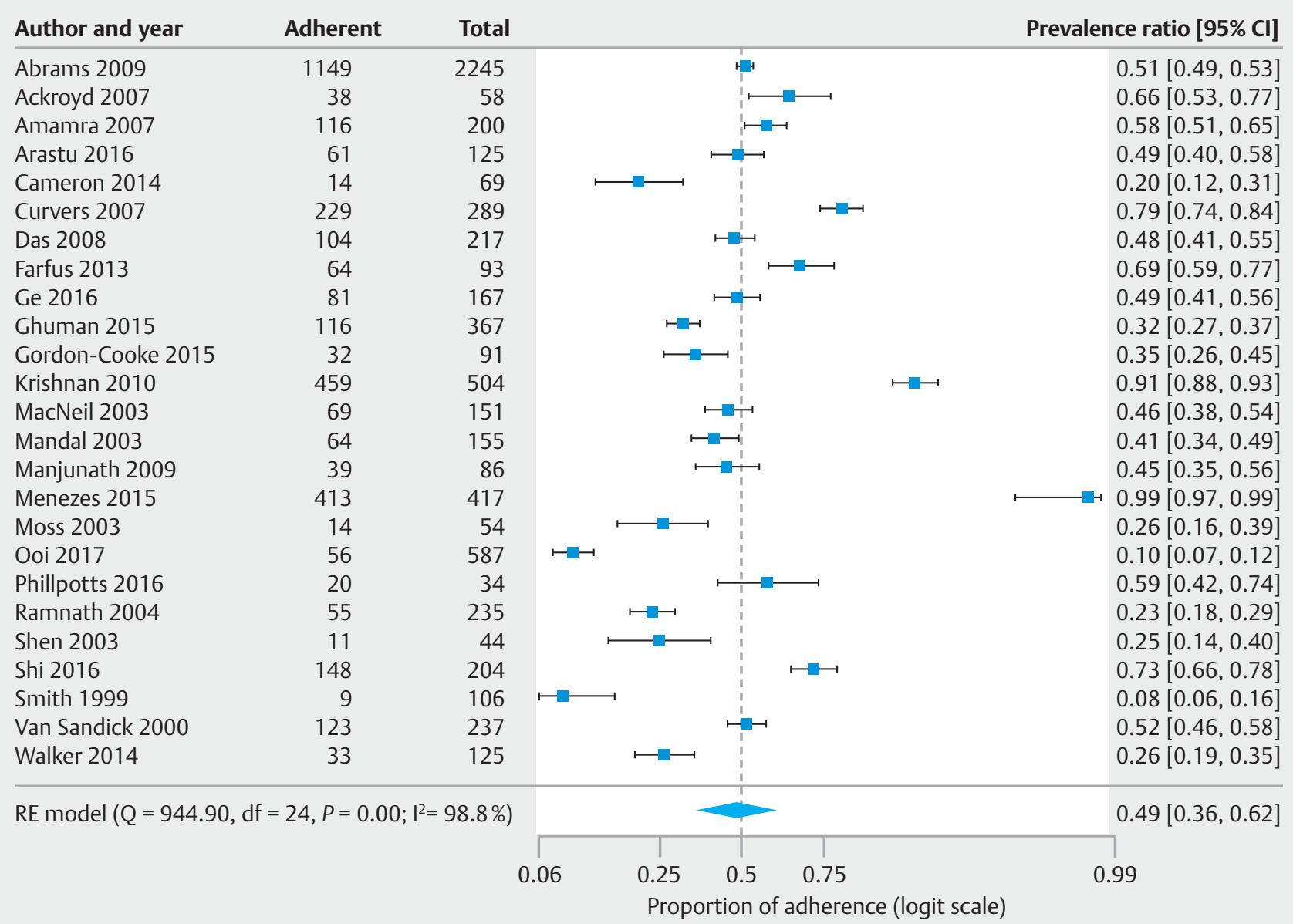

- Fig. 3 Forest plot of pooled proportion of adherence to guideline recommendation "Seattle protocol." Cl, confidence interval; RE, random effect.

\section{Discussion}

In this systematic review and meta-analysis, we pooled the overall worldwide rates of adherence to guideline recommendations as researched in the literature, which showed a large variance between studies. Difference in country and type of practice were the main contributing factors for the large heterogeneity in pooled estimates. Although many studies aimed to identify explanatory parameters for nonadherence, most of them were observed in univariate analysis in a retrospective cohort design. The graphical representation of this study shows that factors most frequently reported to be associated with better adherence were shorter BE length, salaried employment, surveillance in university hospitals, and dedicated surveillance programs. We performed our research according to established guidelines for systematic reviews and did a thorough meta-analysis. Because results from multiple studies were combined, this study provides insight into the variability between studies, rather than adherence rates of single studies.

In line with the subgroup analysis, surveillance was reported to be more often in line with guideline recommendations in university hospitals than in general hospitals. In addition, bet- ter adherence was observed in dedicated programs. This indicates that persistent alertness of guidelines is important. We found that interventions such as a dedicated nurse and other formalized surveillance programs improved adherence, and that the effect of only implementing a guideline is limited. It is known that more than 1 year after a guideline has been implemented, clinicians' adherence to it declines [32]. Simply being aware of the guideline was not often contributing to adherence in our review; however, younger gastroenterologists, who had been trained more recently, were more adherent to the use of the Prague criteria. University hospitals, with their emphasis on teaching and research, were also associated with better adherence.

Remarkably, we found that more intensive surveillance was recommended if there was more belief in the efficacy of surveillance, and that more agreement with the guideline was related to better adherence to the surveillance interval. Similar findings have been reported for adherence to guidelines in other specialties [33]. Furthermore, the evidence behind the Barrett's surveillance guideline recommendations is moderate at best. Therefore, to improve adherence, the evidence underpinning 


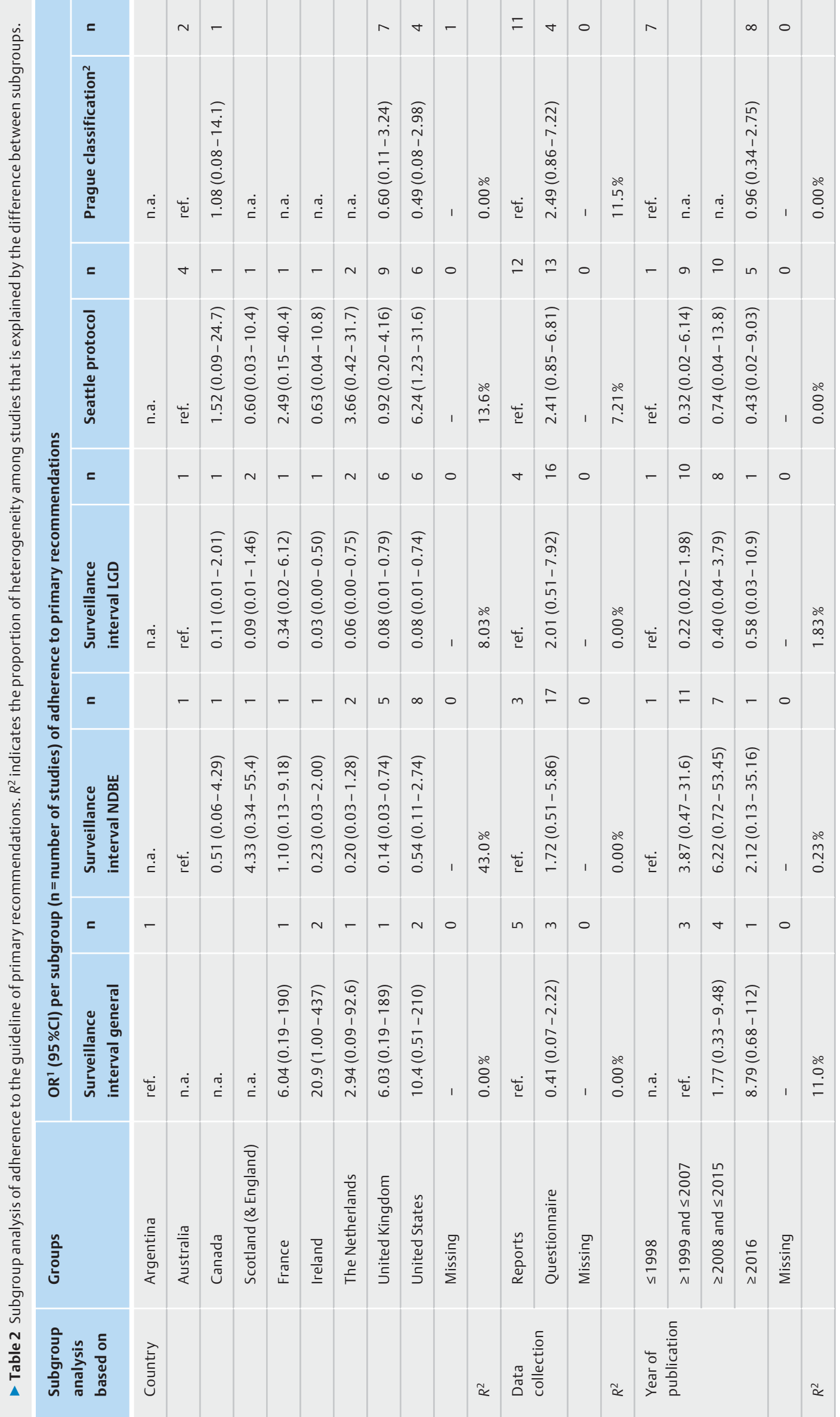




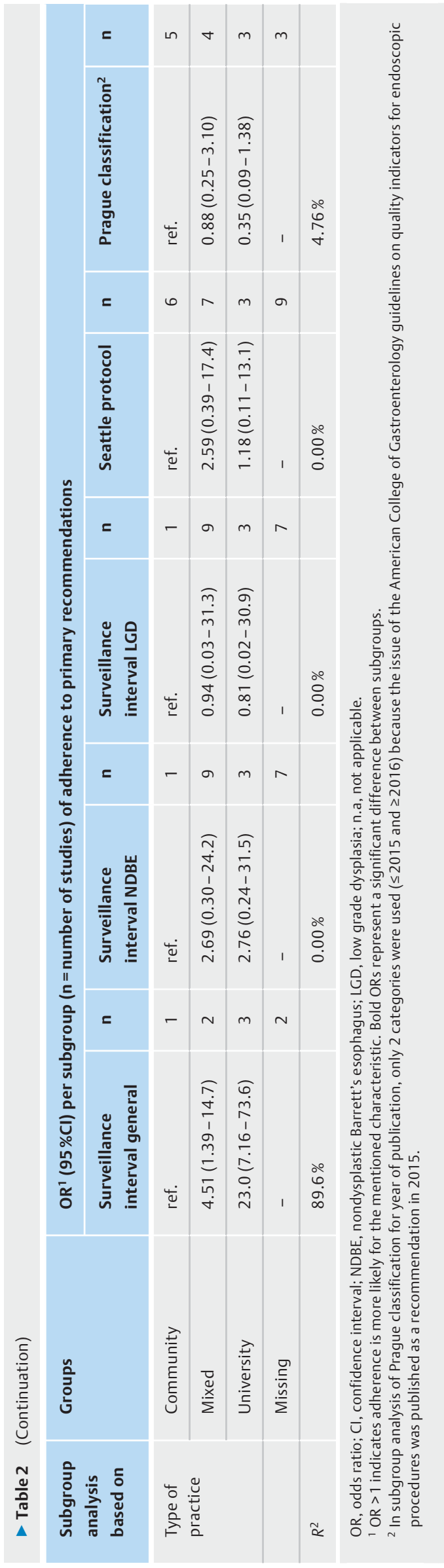

the guidelines should be strengthened, for example by improvement of risk stratification.

In this systematic review, pooled estimates showed large heterogeneity, as expected. To evaluate the influence of methodological differences of studies, rather than, for example, type of practice, we also estimated whether the data could be influenced by self-reported data collection [34]. This explained only a small proportion of the heterogeneity in the subgroup analysis. However, as the heterogeneity between studies addressing adherence to Prague classification could be explained by $>10 \%$ by differences in data collection, results should be interpreted with caution. We also estimated the influence of the year of publication. In other words, we evaluated whether the year of publication could explain heterogeneity, and if adherence improves or declines over time. For surveillance interval in general, $>10 \%$ of heterogeneity could be explained by the year of publication. Although the results per year category are not statistically significant, increasing ORs for categories of more recent publications may indicate improving adherence over the years.

To our knowledge, this is the first study to provide the pooled estimates of adherence to guideline recommendations of BE surveillance. The findings of this study stress the relevance of improving the adherence of BE surveillance for two reasons. First, poor adherence to the Seattle protocol could increase the health care burden. Although there is no conclusive evidence available, the Seattle protocol has been shown to reduce sampling error with improved detection of dysplasia, compared with random biopsies [35]. Alternatively, nonadherence reduces the likelihood of detecting dysplasia. In a previous study with a cohort of 2245 BE patients under surveillance, the detection was reduced by almost half in cases of nonadherence to the biopsy protocol [7]. Consequently, because of nonadherence, EAC may be detected at a later stage, with a negative influence on the outcome for BE patients. However, missed dysplasia in a repeat endoscopy within 24 months was not associated with adherence to the Seattle protocol [36]. The detection of dysplasia was influenced not only by adherence to the biopsy protocol, but adherence to reporting the length of the BE segment using the Prague classification was also associated with better detection of dysplasia [37].

Second, performing surveillance endoscopies at an earlier time point than that recommended in the guideline increases the total number of surveillance endoscopies performed. The cost-effectiveness of the current surveillance strategies, as recommended by guidelines, is under discussion; there is disagreement about the optimal surveillance interval. However, three studies suggested this interval should not be less than 3 years for NDBE and not less than 1 year for $\operatorname{LGD}[9,38]$. The results of these studies indicate that a shorter interval than recommended by the current guidelines would increase the health care costs even more. Only one study reported cost-effectiveness with shorter intervals [39].

Consequently, if impaired cost-effectiveness of surveillance due to shorter surveillance intervals is accompanied by lower detection of dysplasia because of nonadherence to the Seattle protocol and the Prague classification, the health care burden 


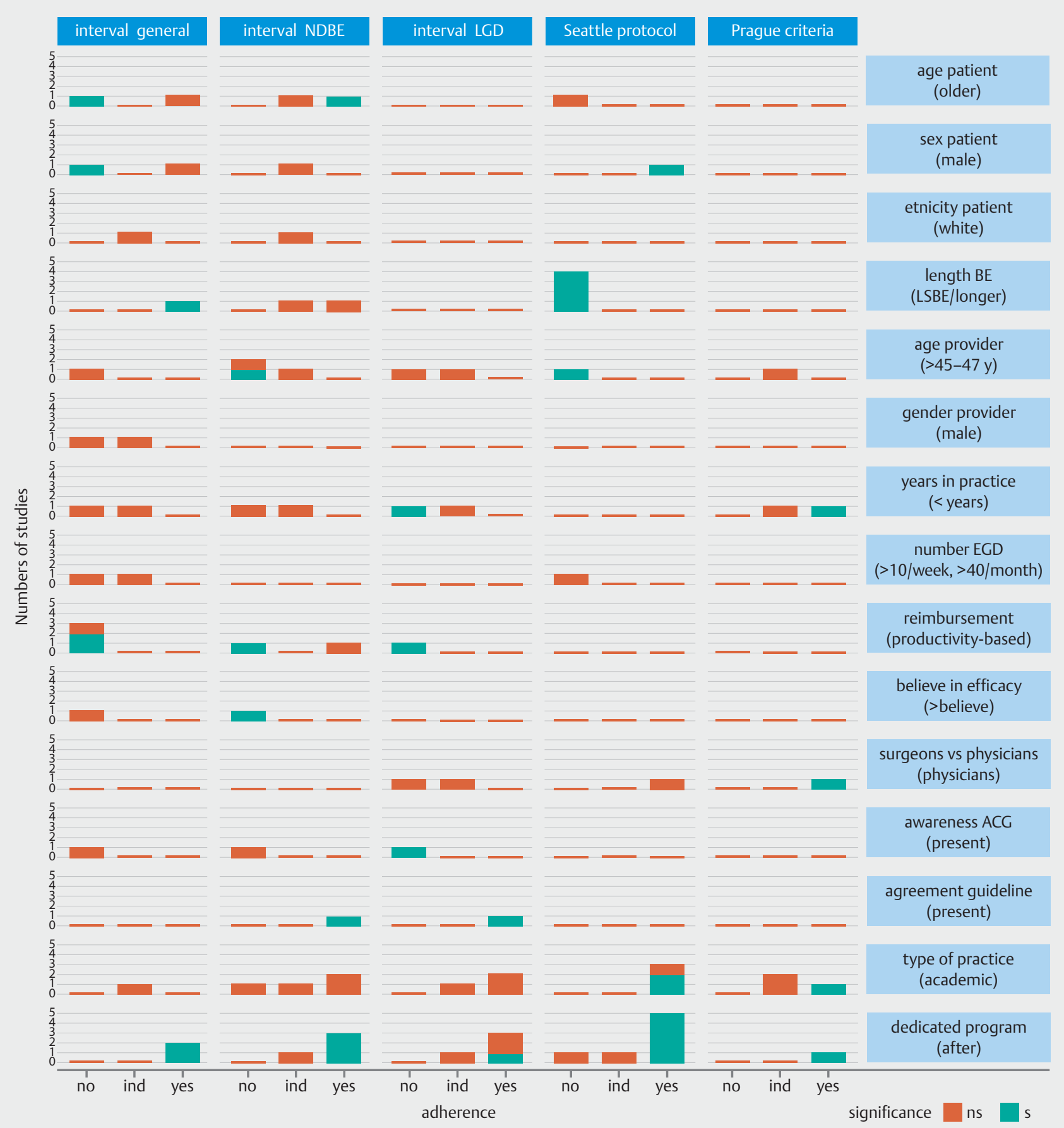

- Fig. 4 Overview of association between primary recommendations of adherence to the guideline (top horizontal pane) and explanatory parameters (right vertical pane). The $x$-axis (adherence) represents whether studies were associated with adherence ("yes"), not associated with adherence ("no"), no difference between groups ("ind", if the absolute proportions of each group were not mentioned in the study, only that there was no difference). These results could be significant (black, "s"), or nonsignificant (grey, "ns"). The $y$-axis represents number of studies that reported the associations as mentioned in the graph. Results of multivariate analysis were used if available. ACG, American College of Gastroenterology; BE, Barrett's esophagus; EGD, esophagogastroduodenoscopy; LGD, low grade dysplasia; LSBE, long-segment Barrett's esophagus; NDBE, nondysplastic Barrett's esophagus.

of surveillance of BE patients is disproportionate. This results in exorbitant costs for society and poorer detection of early neoplastic progression in BE patients.
This study has several strengths and limitations. We conducted this systematic review and meta-analysis according to standardized protocols. Additional analysis for between-study variation provided insight into data and heterogeneity of re- 
sults. The methodological quality of some included studies was limited, with data collection based on self-report as a contributing factor in over one-third of articles. As all studies on adherence have been conducted in highly developed countries, caution should be exercised with regard to the generalizability of the results. An important limitation with a potentially large contribution to the results is that parameters associated with adherence were identified in most studies in univariate analysis, whereas other confounders could be important as well.

In conclusion, adherence to BE surveillance guidelines is suboptimal, thereby adversely affecting health care burden (e.g. impaired detection of dysplasia and superfluous costs). Opportunities for improving adherence should be further investigated, preferably without self-reported surveys and with adjustment for confounding factors. Attention should be paid to increase the evidence for guideline recommendations. Shortterm meaningful consequences include optimal introduction and monitoring of formalized surveillance programs.

\section{Acknowledgments}

We would like to kindly acknowledge Gerdien de Jonge, biomedical information specialist, Erasmus University Rotterdam, who was of great help in the literature search for this study.

\section{Competing interests}

None

\section{References}

[1] Rice TW, Blackstone EH, Goldblum JR et al. Superficial adenocarcinoma of the esophagus. J Thorac Cardiovasc Surg 2001; 122: 1077 1090

[2] van Sandick JW, van Lanschot JJ, Kuiken BW et al. Impact of endoscopic biopsy surveillance of Barrett's oesophagus on pathological stage and clinical outcome of Barrett's carcinoma. Gut 1998; 43: $216-222$

[3] Fitzgerald RC, di Pietro M, Ragunath K et al. British Society of Gastroenterology guidelines on the diagnosis and management of Barrett's oesophagus. Gut 2014; 63: 7-42

[4] Shaheen NJ, Falk GW, lyer PG et al. ACG Clinical Guideline: Diagnosis and management of Barrett's esophagus. Am J Gastroenterol 2016; 111: $30-50$

[5] Spechler SJ, Sharma P, Souza RF. American Gastroenterological Association. et al. American Gastroenterological Association medical position statement on the management of Barrett's esophagus. Gastroenterology 2011; 140: 1084-1091

[6] Woolf SH, Grol R, Hutchinson A et al. Potential benefits, limitations and harms of clinical guidelines. BMJ 1999; 318: 527-530

[7] Abrams JA, Kapel RC, Lindberg GM et al. Adherence to biopsy guidelines for Barrett's esophagus surveillance in the community setting in the United States. Clin Gastroenterol Hepatol 2009; 7: 736-742

[8] Peters FP, Curvers WL, Rosmolen WD et al. Surveillance history of endoscopically treated patients with early Barrett's neoplasia: nonadherence to the Seattle biopsy protocol leads to sampling error. Dis Esophagus 2008; 21: $475-479$
[9] Kastelein F, van Olphen S, Steyerberg EW et al. Surveillance in patients with long-segment Barrett's oesophagus: a cost-effectiveness analysis. Gut 2015; 64: $864-871$

[10] Menezes A, Tierney A, Yang YX et al. Adherence to the 2011 American Gastroenterological Association medical position statement for the diagnosis and management of Barrett's esophagus. Dis Esophagus 2015; 28: 538- 546

[11] Sharma P, Dent J, Armstrong D et al. The development and validation of an endoscopic grading system for Barrett's esophagus: the Prague C \& M criteria. Gastroenterology 2006; 131: 1392 -1399

[12] American Society for Gastrointestinal Endoscopy. The role of endoscopy in the surveillance of premalignant conditions of the upper gastrointestinal tract. Gastrointest Endosc 1998; 48: 663-668

[13] Evans JA, Early DS. ASGE Standards of Practice Committee. et al. The role of endoscopy in Barrett's esophagus and other premalignant conditions of the esophagus. Gastrointest Endosc 2012; 76: 1087 1094

[14] Beck IT, Champion MC, Lemire S et al. The Second Canadian Consensus Conference on the Management of Patients with Gastroesophageal Reflux Disease. Can J Gastroenterol 1997; 11: 7B-20B

[15] Boyer J, Laugier R, Chemali M et al. French Society of Digestive Endoscopy SFED guideline: Monitoring of patients with Barrett's esophagus. Endoscopy 2007; 39: 840-842

[16] Boyer J, Robaszkiewicz M. Guidelines of the French Society of Digestive Endoscopy: Monitoring of Barrett's esophagus. The Council of the French Society of Digestive Endoscopy. Endoscopy 2000; 32: 498 499

[17] Cohen J, Safdi MA, Deal SE et al. Quality indicators for esophagogastroduodenoscopy. Gastrointest Endosc 2006; 63: S10-15

[18] Dent J, Bremner CG, Collen MJ. Working party report to the World Congresses of Gastroenterology, Sydney 1990; Barrett's esophagus. J Gastroenterol Hepatol 1991; 6: 1-22

[19] di Pietro M, Fitzgerald RC. BSG Barrett's Guidelines Working Group. Revised British Society of Gastroenterology recommendation on the diagnosis and management of Barrett's oesophagus with low-grade dysplasia. Gut 2018; 67: 392-393

[20] Hirota WK, Zuckerman M], Adler DG et al. ASGE guideline: the role of endoscopy in the surveillance of premalignant conditions of the upper Gl tract. Gastrointest Endosc 2006; 63: 570 - 580

[21] Kahrilas PJ, Shaheen NJ, Vaezi MF et al. American Gastroenterological Association Medical Position Statement on the management of gastroesophageal reflux disease. Gastroenterology 2008; 135: 1383 1391

[22] Katelaris P, Holloway R, Talley N et al. Gastro-oesophageal reflux disease in adults: guidelines for clinicians. J Gastroenterol Hepatol 2002; 17: $825-833$

[23] Sampliner RE. Practice guidelines on the diagnosis, surveillance, and therapy of Barrett's esophagus. The Practice Parameters Committee of the American College of Gastroenterology. Am J Gastroenterol 1998; 93: $1028-1032$

[24] Sampliner RE. Practice Parameters Committee of the American College of Gastroenterology. Updated guidelines for the diagnosis, surveillance, and therapy of Barrett's esophagus. Am J Gastroenterol 2002; 97: $1888-1895$

[25] Siersema PD, Bergman JJGHM, Van Berge HenegouwenMl et al. Guideline Barrett's esophagus. article in Dutch 2017: Available from: https://www.mdl.nl/sites/www.mdl.nl/files/richlijnen/Richtlijnen\% 20Barrett\%20oesofagus\%20-\%20jan\%202018\%20-\%20tbv\%20website.pdf

[26] Wang KK, Sampliner RE. Practice Parameters Committee of the American College of Gastroenterology. Updated guidelines 2008 for the diagnosis, surveillance and therapy of Barrett's esophagus. Am J Gastroenterol 2008; 103: 788-797 
[27] Wang KK, Wongkeesong M, Buttar NS et al. American Gastroenterological Association Medical Position Statement: Role of the gastroenterologist in the management of esophageal carcinoma. Gastroenterology 2005; $128: 1468-1470$

[28] Watson A, Heading RC, Shepherd NA. Guidelines for the diagnosis and management of Barrett's columnar-lined oesophagus. A report of the working party of the British Society of Gastroenterology. London: BSG; 2005

[29] Whiteman DC, Appleyard M, Bahin FF et al. Australian clinical practice guidelines for the diagnosis and management of Barrett's esophagus and early esophageal adenocarcinoma. J Gastroenterol Hepatol 2015; 30: $804-820$

[30] Hayden JA, van der Windt DA, Cartwright JL et al. Assessing bias in studies of prognostic factors. Ann Intern Med 2013; 158: 280 - 286

[31] Higgins JP, Thompson SG, Deeks JJ et al. Measuring inconsistency in meta-analyses. BMJ 2003; 327: 557-560

[32] Ament SM, de Groot JJ, Maessen JM et al. Sustainability of professionals' adherence to clinical practice guidelines in medical care: a systematic review. BMJ Open 2015; 5: e008073
[33] Cnossen MC, Scholten AC, Lingsma HF et al. Adherence to guidelines in adult patients with traumatic brain injury: a living systematic review. J Neurotrauma 2016; 33:1-14

[34] Montano DE, Phillips WR. Cancer screening by primary care physicians: a comparison of rates obtained from physician self-report, patient survey, and chart audit. Am J Public Health 1995; 85: $795-800$

[35] Abela JE, Going JJ, Mackenzie JF et al. Systematic four-quadrant biopsy detects Barrett's dysplasia in more patients than nonsystematic biopsy. Am J Gastroenterol 2008; 103: 850-855

[36] Visrodia K, lyer PG, Schleck CD et al. Yield of repeat endoscopy in Barrett's esophagus with no dysplasia and low-grade dysplasia: a population-based study. Dig Dis Sci 2016; 61: 158 - 167

[37] Ghuman S, Ashgar K, Evans J et al. Real world surveillance of Barrett's oesophagus does it make a difference using the Prague classification or following Seattle biopsy protocol? Gut 2015; 64: PTH- 028

[38] Inadomi JM, Somsouk M, Madanick RD et al. A cost-utility analysis of ablative therapy for Barrett's esophagus. Gastroenterology 2009; 136: $2101-2114$

[39] Gordon LG, Mayne GC, Hirst NG et al. Cost-effectiveness of endoscopic surveillance of non-dysplastic Barrett's esophagus. Gastrointest Endosc 2014; 79: 242 -256 